PROCEEDINGS OF THE

AMERICAN MATHEMATICAL SOCIETY

Volume 126, Number 12, December 1998, Pages 3681-3692

S $0002-9939(98) 04985-5$

\title{
POSITIVE SOLUTIONS OF NONLINEAR ELLIPTIC EQUATIONS IN THE EUCLIDEAN PLANE
}

\author{
U. UFUKTEPE AND Z. ZHAO
}

(Communicated by Jeffrey B. Rauch)

\begin{abstract}
In the present paper, we study the existence of solutions to the problem

$$
\left\{\begin{array}{cc}
\Delta u+f(x, u)=0 & \text { in } D \\
u>0 & \text { in } D \\
u=0 & \text { on } \partial D
\end{array}\right.
$$

where $D$ is an unbounded domain in $\mathbb{R}^{2}$ with a compact nonempty boundary $\partial D$ consisting of finitely many Jordan curves. The goal is to prove an existence theorem for the above problem in a general setting by using Brownian path integration and potential theory.
\end{abstract}

\section{INTRODUCTION}

In the present paper, we study the existence of solutions to the problem

$$
\left\{\begin{array}{cc}
\Delta u+f(x, u)=0 & \text { in } D \\
u>0 & \text { in } D \\
u=0 & \text { on } \partial D
\end{array}\right.
$$

where $D$ is an unbounded domain in $\mathbb{R}^{2}$ with a compact nonempty boundary $\partial D$ consisting of finitely many Jordan curves. The goal is to prove an existence theorem for problem (1.1) in a general setting by using Brownian path integration and potential theory.

For $D \subset \mathbb{R}^{n}, n \geq 3, D$ unbounded and with a compact Lipschitz boundary, Z. Zhao [13] proved the following problem:

$$
\left\{\begin{array}{cc}
\Delta u+K(x) f(u)=0 & \text { in } D \\
u>0 & \text { in } D \\
u=0 & \text { on } \partial D
\end{array}\right.
$$

if $K$ is a Borel measurable function in $D$ satisfying that the family $\left\{\frac{K(.)}{|\cdot-x| d-2}\right\}$ is uniformly integrable over $D$ with a parameter $x \in D$, and $f$ is a continuous function in $(0, b)$ for some $0<b \leq \infty$ satisfying that:

$$
\lim _{w \rightarrow 0^{+}} \frac{f(w)}{w}=0
$$

Received by the editors March 10, 1997.

1991 Mathematics Subject Classification. Primary 60J45, 60J65.

(c)1998 American Mathematical Society 
Then the problem (1.2) has infinitely many bounded solutions. More precisely, there exists $b_{0} \in(0, b]$ such that for each $c \in\left(0, b_{0}\right]$ there exists a solution $u$ of $(1.2)$ satisfying

$$
\lim _{|x| \rightarrow \infty} u(x)=c .
$$

A similar nonlinear problem for the second order ordinary differential equations is studied in [14]. We state our main result as

Theorem 1.1 (The Main Theorem). Let $D$ be an unbounded domain in $\mathbb{R}^{2}$ with a compact nonempty boundary $\partial D$ consisting of finitely many Jordan curves. Suppose that $f(x, s)$ is a Borel measurable function in $\mathbb{R}^{2} \times \mathbb{R}^{+}$and $f(x, \cdot)$ is a continuous function in $\mathbb{R}^{+}$for each fixed $x \in \mathbb{R}^{2}$. If there exists a positive, convex and continuously differentiable function $F(x, s)$ in $\mathbb{R}^{2} \times \mathbb{R}^{+}$satisfying the conditions

$$
\begin{gathered}
|f(x, s)| \leq F(x, s), \quad(x, s) \in \mathbb{R}^{2} \times \mathbb{R}^{+}, \\
F(x, 0)=F_{s}(x, 0)=0
\end{gathered}
$$

and

$$
F_{s}(x, \ln (|x|+1)) \in K_{2}^{\infty},
$$

then the problem

$$
\left\{\begin{array}{cc}
\Delta u+f(x, u)=0 & \text { in } D \\
u>0 & \text { in } D \\
u=0 & \text { on } \partial D
\end{array}\right.
$$

has infinitely many solutions. More precisely there exists a number $b>0$ such that for each $c \in(0, b],(1.6)$ has a solution u satisfying

$$
\lim _{|x| \rightarrow \infty} \frac{u(x)}{\ln |x|}=c .
$$

Remark. A special case of the above general setting is the semilinear problem for any $V$ in $K_{2}$, and $p>1$ : If we take $f(x, s)=V(x) s^{p}$ and $F(x, s)=|V(x)| s^{p}$, then problem (1.1) becomes the corresponding problem for the semilinear elliptic equation

$$
\left\{\begin{array}{cc}
\Delta u+V(x) u^{p}=0 & \text { in } D \\
u>0 & \text { in } D \\
u=0 & \text { on } \partial D .
\end{array}\right.
$$

Then $f$ and $F$ satisfy (1.3) and (1.4) obviously. Condition (1.5) on $F$ is equivalent to

$$
\int_{|x| \geq 1}|V(x)|[\ln (1+|x|)]^{p} d x<\infty .
$$

\section{Green functions and $h$-Conditional Brownian motion}

In this section we collect the results on Green functions in $\mathbb{R}^{2}$ and $h$-conditional Brownian motion. 
The Kato class $K_{2}$ is defined to be the set of all Borel measurable functions on $\mathbb{R}^{2}$ satisfying

$$
\lim _{\alpha \downarrow 0}\left[\sup _{x \in \mathbb{R}^{2}} \int_{|y-x|<\alpha} \ln \frac{1}{|y-x|}|q(y)| d y\right]=0
$$

where $q: \mathbb{R}^{2} \rightarrow \mathbb{R}$ is measurable. $K_{2}$ is an important class of the potential functions for the Schrödinger operator. Another potential class $K_{2}^{\infty}$ which was introduced in $[5]$ is as follows:

$$
K_{2}^{\infty}=\left\{q \in K_{2}: \int_{|y|>1} \ln |y||q(y)| d y<\infty\right\} .
$$

Let $\left\{X_{t}\right\}$ be 2-dimensional Brownian motion (see [2]). For any open or closed set $A$ in $\mathbb{R}^{2}$, let

$$
T_{A}(\omega)=\inf \left\{t>0: X_{t}(\omega) \in A\right\} .
$$

This is called the hitting time of $A$. The hitting time of $A^{c}$ is called the exit time from $A$ and denoted by $\tau_{A}$. We shall use $P^{x}$ to denote the probability measure on the Brownian continuous paths starting at $x$. $E^{x}$ is the expectation on $P^{x}$. Let $G_{D}(x, y)$ be the Green function for $D$. We list some known results.

For any Borel function $\varphi$ in $D$ the Green operator is defined as

$$
\begin{aligned}
G_{D} \varphi(x) & =E^{x}\left[\int_{0}^{\tau_{D}} \varphi\left(X_{t}\right) d t\right] \\
& =\int_{D} G_{D}(x, y) \varphi(y) d y,
\end{aligned}
$$

and

$$
\Delta\left(G_{D} \varphi\right)=-2 \varphi .
$$

Proposition 2.1. Let $D$ be an unbounded domain in $\mathbb{R}^{2}$ with a compact nonempty boundary $\partial D$ consisting of finitely many Jordan curves. Then there exists a harmonic function $h>0$ in $D$ such that

$$
\lim _{D \ni x \rightarrow z} h(x)=0 \quad \forall z \in \partial D,
$$

and

$$
\lim _{|x| \rightarrow \infty} \frac{h(x)}{\ln |x|}=1 .
$$

Proof. Pick a point $a \in \mathbb{R}^{2} \backslash \bar{D}$ and $r>0$ (small enough) such that $\bar{B}(a, r) \subset \mathbb{R}^{2} \backslash \bar{D}$. Let $x^{*}=a+r^{2} \frac{(x-a)}{|x-a|^{2}}$ be the Kelvin inversion from $D \cup\{\infty\}$ onto $D^{*}$, where $D^{*}=\left\{x^{*} \in B(a, r): x \in D \cup\{\infty\}\right\}$. Since the Kelvin inversion preserves harmonic functions and $D^{*}$ is bounded Jordan domain, we have

$$
G_{D}(x, y)=G_{D^{*}}\left(x^{*}, y^{*}\right), \quad \forall x, y \text { in } D .
$$

Let

$$
h(x)=\pi G_{D^{*}}\left(x^{*}, a\right) .
$$


Thus, we have

$$
\lim _{y \rightarrow \infty} \pi G_{D}(x, y)=\lim _{y^{*} \rightarrow a} \pi G_{D^{*}}\left(x^{*}, y^{*}\right)=\pi G_{D^{*}}\left(x^{*}, a\right) .
$$

The rest of the proof is just the properties of the Green function in the bounded domain.

Now we can develop the notion of conditional Brownian motion introduced by Doob for a general theory. Let $h>0$ be a harmonic function in $D$. We define

$$
p_{h}^{D}(t ; x, y)=h(x)^{-1} p^{D}(t ; x, y) h(y), \quad t>0, \quad x, y \in D,
$$

where $p^{D}$ is the transition density of the killed Brownian motion. $p_{h}^{D}(t ; x, y)$ satisfies the conditions for a transition density. Let $P_{h}^{x}$ and $E_{h}^{x}$ denote respectively the probability and expectation determined by the $h$-conditional Brownian motion starting at $x$. Then we have for all $f \in \mathcal{B}^{+}(D)$ :

$$
E_{h}^{x}\left[t<\tau_{D} ; f\left(X_{t}\right)\right]=h(x)^{-1} E^{x}\left[t<\tau_{D} ; f\left(X_{t}\right) h\left(X_{t}\right)\right], \quad x \in D .
$$

Theorem 2.2 (3-G). Let $D \subset \mathbb{R}^{2}$ be as in Proposition 2.1. Then there exists a constant $\mathbf{C}>0$ depending on $D$ only such that $\forall x, y$, and $z \in D$ :

$$
\frac{G_{D}(x, y) G_{D}(y, z)}{G_{D}(x, z)} \leq \mathbf{C}\left[G_{D}(x, y)+G_{D}(y, z)+1\right] .
$$

Proof. We used Kelvin transformation to reduce to the bounded Jordan domain case (see [2]). For $n \geq 3$, the 3 -G theorem for the unbounded Lipschitz domain was proved by Herbst and Zhao [6] based on [4].

Proposition 2.3. If $q \in K_{2}^{\infty}$, then $q \in L^{1}\left(\mathbb{R}^{2}\right)$.

Proof. Let $q \in K_{2}^{\infty}$. Then by definition of $K_{2}^{\infty}, q \in K_{2}$. So there exists $\alpha_{1}>0$ such that

$$
\sup _{x \in \mathbb{R}^{2}} \int_{|y-x| \leq \alpha_{1}} \ln \frac{1}{|x-y|}|q(y)| d y<1
$$

and

$$
\int_{|y| \geq e} \ln |y||q(y)| d y<\infty .
$$

Let $\alpha=\min \left(\alpha_{1}, e^{-1}\right)$. For the compact set $\bar{B}=\bar{B}(0, e)$, there exists finite points $x_{i}, i=1, \ldots, L$, such that $\bar{B} \subseteq \bigcup_{i=1}^{L} B\left(x_{i}, \alpha\right)$ and $\forall i=1, \ldots, L, y \in B\left(x_{i}, \alpha\right)$ we have $\ln \frac{1}{\left|x_{i}-y\right|} \geq \ln \frac{1}{e^{-1}}=1$. Then by $(2.8)$

$$
\begin{aligned}
\int_{|y| \leq e}|q(y)| d y & \leq \sum_{i=1}^{L} \int_{B\left(x_{i}, \alpha\right)}|q(y)| d y \\
& \leq \sum_{i=1}^{L} \int_{B\left(x_{i}, \alpha\right)} \ln \frac{1}{\left|x_{i}-y\right|}|q(y)| d y \\
& \leq L .
\end{aligned}
$$


Since $q \in K_{2}^{\infty}$, we have

$$
\int_{|y|>e}|q(y)| d y \leq \int_{|y|>e} \ln |y||q(y)| d y<\infty .
$$

Thus, by (2.10) and (2.11)

$$
\int_{\mathbb{R}^{2}}|q(y)| d y \leq \int_{|y|>e}|q(y)| d y+L<\infty .
$$

\section{UNIFORMLY INTEGRABLE FUNCTIONS}

This is the main technical section in which we investigate uniform integrability of the family of the functions $\left\{G_{D}(x,).|q()|.\right\}$ and $\left\{\frac{1}{h(x)} G_{D}(x,) h.().|q()|.\right\}$.

Since $D \supset B_{r}^{*}=\mathbb{R}^{2} \backslash \overline{B(a, r)}$, using the explicit formula of $G_{B_{r}^{*}}(.,$.$) and the$ definition of $K_{2}^{\infty}$, we can prove:

Proposition 3.1. For $q \in K_{2}^{\infty}$, the family of functions $\left\{G_{D}(x, \cdot)|q(\cdot)|\right\}$ with parameter $x \in D$ is uniformly integrable over $D$.

We define

$$
\|q\|_{D}=\sup _{x \in D} \int_{D} G(x, y)|q(y)| d y
$$

and

$$
\|q\|_{1}=\int_{D}|q(y)| d y
$$

Proposition 3.2. Let $D$ and $h$ be as in Proposition 2.1. Then for any $q \in K_{2}^{\infty}$, we have

(a) $\left\{\frac{1}{h(x)} G_{D}(x, \cdot) h(\cdot)|q(\cdot)|: x \in D\right\}$ is uniformly integrable.

(b) There exists a constant $c_{D}$ such that $\forall x \in D$ :

$$
\frac{1}{h(x)} \int_{D} G_{D}(x, y) h(y)|q(y)| d y \leq c_{D}\left(\|q\|_{D}+\|q\|_{1}\right) .
$$

Proof. By Theorem (3-G), there exists $\mathbf{C}>0$ such that

$$
\frac{G_{D}(x, y) G_{D}(y, z)|q(y)|}{G_{D}(x, z)} \leq \mathbf{C}\left(G_{D}(x, y)+G_{D}(y, z)+1\right)|q(y)| .
$$

Recalling $h(x)=\lim _{z \rightarrow \infty} \pi G_{D}(x, z)$, and using Proposition 3.1 and the fact $q \in$ $L^{1}(D)$, we can obtain that $\left\{\frac{1}{h(x)} G_{D}(x, \cdot) h(\cdot)|q(\cdot)|: x \in D\right\}$ is uniformly integrable. Thus, (a) follows. 
By taking the integral of (3.4) with respect to $y$, we have

$$
\begin{aligned}
& \frac{1}{G_{D}(x, z)} \int_{D} G_{D}(x, y) G_{D}(y, z)|q(y)| d y \\
& \quad \leq \mathbf{C}\left(\int_{D} G_{D}(x, y)|q(y)| d y+\int_{D} G_{D}(y, z)|q(y)| d y+\int_{D}|q(y)| d y\right) \\
& \quad \leq c_{D}\left(\|q\|_{D}+\|q\|_{1}\right),
\end{aligned}
$$

where $c_{D}=2 \mathbf{C}$. By taking the limit of (3.5) as $z \rightarrow \infty$, we have by Fatou's lemma:

$$
\frac{1}{h(x)} \int_{D} G_{D}(x, y) h(y)|q(y)| d y \leq c_{D}\left(\|q\|_{D}+\|q\|_{1}\right) .
$$

Proposition 3.3. Let $D$ and $h$ be as in Proposition 2.1. Let $q \in K_{2}^{\infty}$. If

$$
\sup _{x \in D} E_{h}^{x}\left[\int_{0}^{\tau_{D}}\left|q\left(X_{t}\right)\right| d t\right] \leq \frac{1}{2}
$$

then the function

$$
w(x)=h(x) E_{h}^{x}\left[e^{\int_{0}^{\tau} D} q\left(X_{s}\right) d s\right], \quad \forall x \in D,
$$

is well defined in $D$ and satisfies that $\forall x \in D$

$$
e^{-\frac{1}{2}} h(x) \leq w(x) \leq 2 h(x)
$$

and

$$
w(x)=h(x)+\int_{D} G_{D}(x, y) q(y) w(y) d y .
$$

Proof. By Khas'minskii's lemma, we have by (3.6),

$$
E_{h}^{x}\left[e^{\int_{0}^{\tau} D q\left(X_{s}\right) d s}\right] \leq 2
$$

By Jensen's inequality and (3.6), we have

$$
e^{-\frac{1}{2}} \leq E_{h}^{x}\left[e^{\int_{0}^{\tau} D} q\left(X_{s}\right) d s\right] .
$$

Thus, it follows by (3.7), (3.10), and (3.11) that

$$
h(x) e^{-\frac{1}{2}} \leq w(x) \leq 2 h(x) .
$$


The equality (3.9) will be obtained by calculation on the conditional Brownian paths:

$$
\begin{aligned}
& w(x)-h(x)=h(x) E_{h}^{x}\left[e^{\int_{0}^{\tau} D} q\left(X_{s}\right) d s-1\right] \\
& =h(x) E_{h}^{x}\left[\int_{0}^{\tau_{D}} q\left(X_{t}\right) e^{\int_{t}^{\tau_{D} D} q\left(X_{s}\right) d s} d t\right] \\
& =h(x) \int_{0}^{\infty} E_{h}^{x}\left[t<\tau_{D}: q\left(X_{t}\right) e^{\int_{t}^{\tau_{t}} q\left(X_{s}\right) d s}\right] d t \\
& =h(x) \int_{0}^{\infty} E_{h}^{x}\left[t<\tau_{D}: q\left(X_{t}\right) E_{h}^{X_{t}}\left[e^{\int_{0}^{\tau_{0}} q\left(X_{s}\right) d s}\right]\right] d t
\end{aligned}
$$

(by the Markov property for the conditional Brownian motion)

$$
\begin{aligned}
& =\int_{0}^{\infty} E^{x}\left[t<\tau_{D} ; q\left(X_{t}\right) h\left(X_{t}\right) E_{h}^{X_{t}}\left[e^{\int_{0}^{\tau_{0}} q\left(X_{s}\right) d s}\right]\right] d t \\
& =\int_{0}^{\infty} E^{x}\left[t<\tau_{D} ; q\left(X_{t}\right) w\left(X_{t}\right)\right] d t \\
& =E^{x}\left[\int_{0}^{\tau_{D}} q\left(X_{t}\right) w\left(X_{t}\right) d t\right] \\
& =\int_{D} G_{D}(x, y) q(y) w(y) d y .
\end{aligned}
$$

\section{Proof of the Main Theorem}

Let $D^{\infty}=\bar{D} \cup\{\infty\}$ be the compactification of $D$. Let

$$
\mathcal{P}(D)=\left\{\psi \in C(D): \lim _{x \rightarrow z \in \partial D} \psi(x) \text { and } \lim _{|x| \rightarrow \infty} \psi(x) \quad \text { exist and are finite }\right\} .
$$

Obviously $\mathcal{P}(D)$ is isometric to $C\left(D^{\infty}\right) . \mathcal{P}(D)$ is a Banach space, with the norm

$$
\|\psi\|=\sup _{x \in D}|\psi(x)| .
$$

Let $q_{0}$ be a positive function belonging to $K_{2}^{\infty}$, and let

$$
Q_{0}=\left\{q \in K_{2}^{\infty}:|q(x)| \leq q_{0}(x), \forall x \in D\right\} .
$$

Proposition 4.1. For a fixed $q_{0}>0$ and $q_{0} \in K_{2}^{\infty}$, and the harmonic function $h$ as defined in Proposition 2.1, the family of the functions

$$
G_{D}^{h}\left[Q_{0}\right]=\left\{\frac{1}{h(\cdot)} \int_{D} G_{D}(\cdot, y) h(y) q(y) d y: q \in Q_{0}\right\}
$$

is uniformly bounded and equicontinuous in $D$, and consequently it is relatively compact in $\mathcal{P}(D)$.

Remark. In order to imply the relative compactness of the family in the supremum norm, the equicontinuity of the family in $\mathcal{P}(D)$ should include that the two limits in (4.1) converge uniformly for all functions in the family. This is a modification of the classical Ascoli-Arzelà theorem. 
Proof. The uniform integrability of $\left\{\frac{1}{h(x)} G(x, \cdot) h(\cdot)|q(\cdot)|: x \in D\right\}$, by Proposition 3.2 , justifies the interchange of the limit and integration, hence we obtain that the function

$$
L_{q}(x) \equiv \frac{1}{h(x)} \int_{D} G_{D}(x, y) h(y) q(y) d y
$$

is continuous. $\lim _{|x| \rightarrow \infty} L_{q}(x)$ and $\lim _{x \rightarrow z \in \partial D} L_{q}(x)$ exist and are finite. Thus, $L_{q}$ belongs to $\mathcal{P}(D)$. For any $q \in Q_{0}$, by (4.2) and 3.2 we have

$$
\begin{aligned}
\sup _{x \in D} \int_{D} \frac{G_{D}(x, y)}{h(x)} h(y)|q(y)| d y & \leq \sup _{x \in D} \int_{D} \frac{G_{D}(x, y)}{h(x)} h(y) q_{0}(y) d y \\
& \leq c_{D}\left(\left\|q_{0}\right\|_{D}+\left\|q_{0}\right\|_{1}\right) .
\end{aligned}
$$

Thus, $G_{D}^{h}\left[Q_{0}\right]$ is uniformly bounded. By using the uniform integrability of $\left\{\frac{1}{h(x)} G_{D}(x, \cdot) h(\cdot) q_{0}(\cdot)\right\}$, we have

i) $\forall q \in Q_{0}, \forall z \in \bar{D}$, as $x, x^{\prime} \rightarrow z$ :

$$
\begin{array}{r}
\left|\int_{D} \frac{G_{D}(x, y)}{h(x)} h(y) q(y) d y-\int_{D} \frac{G_{D}\left(x^{\prime}, y\right)}{h\left(x^{\prime}\right)} h(y) q(y) d y\right| \\
\leq \int_{D}\left|\frac{G_{D}(x, y)}{h(x)}-\frac{G_{D}\left(x^{\prime}, y\right)}{h\left(x^{\prime}\right)}\right| h(y)|q(y)| d y \\
\leq \int_{D}\left|\frac{G_{D}(x, y)}{h(x)}-\frac{G_{D}\left(x^{\prime}, y\right)}{h\left(x^{\prime}\right)}\right| h(y) q_{0}(y) d y \rightarrow 0,
\end{array}
$$

since $x-x^{\prime} \rightarrow 0$.

ii) $\forall q \in Q_{0}$,

$$
\int_{D} \frac{G_{D}(x, y)}{h(x)} h(y)|q(y)| d y \leq \int_{D} \frac{G_{D}(x, y)}{h(x)} h(y) q_{0}(y) d y \rightarrow 0
$$

as $|x| \rightarrow \infty$.

Thus, $G_{D}^{h}\left[Q_{0}\right]$ is equicontinuous in $\mathcal{P}(D)$, and the limits in (4.1) converge uniformly for all functions in $G_{D}^{h}\left[Q_{0}\right]$. Then by the Ascoli-Arzelà theorem $G_{D}^{h}\left[Q_{0}\right]$ is relatively compact in $\mathcal{P}(D)$.

Proof of the Main Theorem. Let $b>0$ be the real number determined later and, for $c \in(0, b]$, let

$$
\Lambda=\left\{v \in \mathcal{P}(D): c e^{-\frac{1}{2}} \leq v(x) \leq 2 c, \quad \forall x \in D\right\} .
$$

For each $v \in \Lambda$ define

$$
T v(x)=c E_{h}^{x}\left[e^{\int_{0}^{\tau} D} q_{v}\left(X_{t}\right) d t\right], \quad \forall x \in D,
$$


where

$$
q_{v}(x)=\frac{f(x, v(x) h(x))}{2 v(x) h(x)} .
$$

For each $x \in D$, by using condition (1.3), (1.4) and the mean value theorem we showed that

$$
\left|\frac{f(x, v(x) h(x))}{2 v(x) h(x)}\right| \leq F_{s}(x, 2 c \eta \ln (|x|+1)) .
$$

We now have

$$
\begin{aligned}
E_{h}^{x}\left[\int_{0}^{\tau_{D}}\left|q_{v}\left(X_{t}\right)\right| d t\right] & =\frac{1}{h(x)} E^{x}\left[\int_{0}^{\tau_{D}} \frac{h\left(X_{t}\right)\left|f\left(X_{t}, v\left(X_{t}\right) h\left(X_{t}\right)\right)\right|}{2 v\left(X_{t}\right) h\left(X_{t}\right)} d t\right] \\
& =\frac{1}{h(x)} \int_{D} \frac{G_{D}(x, y) h(y)|f(y, v(y) h(y))|}{2 v(y) h(y)} d y
\end{aligned}
$$

(by (4.7) and the 3-G Theorem)

$$
\leq \mathbf{C} \int_{D}\left(G_{D}(x, y)+h(y)+1\right) F_{s}(y, 2 c \eta \ln (|y|+1)) d y .
$$

Since $F_{s}(y, \ln (|y|+1)) \in K_{2}^{\infty} \subseteq K_{2}$, there exists $\alpha \in(0,1)$ such that

$$
\sup _{x \in D} \frac{1}{\pi} \int_{|y-x|<\alpha} \ln \frac{1}{|y-x|} F_{s}(y, \ln (|y|+1)) d y<\frac{1}{4 \mathbf{C}} .
$$

By using $F_{s}(y, \ln (|y|+1)) \in K_{2}^{\infty}$ again, we have by Proposition 2.3

$$
\left\{h(y)+1+\frac{1}{\pi}\left[C_{r}+\ln \frac{1}{\alpha}+2 \ln |y-a|\right]\right\} F_{s}(y, \ln (|y|+1)) \in L^{1}(D) .
$$

Thus, using the monotone convergence theorem and noting that $F_{s}(y, 0)=0$ and that $F_{s}(y, \cdot)$ is a nondecreasing function, there exists $\gamma \in(0,1)$ such that

$$
\int_{D}\left[h(y)+1+\frac{1}{\pi}\left(C_{r}+\ln \frac{1}{\alpha}+2 \ln |y-a|\right)\right] F_{s}(y, \gamma \ln (|y|+1)) d y<\frac{1}{4 \mathbf{C}}
$$

Now, we determine $b$ by letting

$$
b=\frac{\gamma}{2 \eta} .
$$

Thus, $\forall x \in D$, for $c \in(0, b], 2 \eta c \leq \gamma<1$, we have

$$
\begin{gathered}
\int_{D} G_{D}(x, y) F_{s}(y, 2 \eta c \ln (|y|+1)) d y \leq \int_{D} G_{B_{r}^{*}(a)}(x, y) F_{s}(y, \gamma \ln (|y|+1)) d y \\
\leq \frac{1}{\pi} \int_{D}\left(C_{r}+2 \ln |y-a|+\ln |x-y|^{-1}\right) F_{s}(y, \gamma \ln (|y|+1)) d y
\end{gathered}
$$

by $(4.9)$

$$
\leq \frac{1}{4 \mathbf{C}}+\frac{1}{\pi} \int_{D}\left(C_{r}+2 \ln |y-a|+\ln \frac{1}{\alpha}\right) F_{s}(y, \gamma \ln (|y|+1)) d y .
$$


It follows from (4.8), (4.10), and (4.12) that

$$
\sup _{x \in D} E_{h}^{x}\left[\int_{0}^{\tau_{D}}\left|q_{v}\left(X_{t}\right)\right| d t\right] \leq \frac{1}{2} .
$$

Similarly,

$$
\sup _{x \in D} E_{h}^{x}\left[\int_{0}^{\tau_{D}} F_{s}\left(X_{t}, 2 c \eta \ln \left(\left|x_{t}\right|+1\right)\right)\right] \leq \frac{1}{2} .
$$

Thus by (4.5), (4.13) and Proposition 3.3 we have

$$
c e^{-\frac{1}{2}} \leq T v(x) \leq 2 c,
$$

and

$$
T v(x)=c+\frac{1}{h(x)} \int_{D} G_{D}(x, y) h(y) q_{v}(y) T v(y) d y .
$$

By (4.15) and the uniform integrability of $\left\{\frac{1}{h(x)} G_{D}(x, \cdot) h(\cdot) q(\cdot): x \in D\right\}$, we see that $\lim _{x \rightarrow z \in \partial D} T v(x)$ and $\lim _{|x| \rightarrow \infty} T v(x)$ exist and are finite. $T v$ is continuous in $D$. Thus, $T v \in \mathcal{P}(D)$ and $T v \in \Lambda$, so

$$
T \Lambda \subseteq \Lambda .
$$

For any $v \in \Lambda$, by (4.7):

$$
\left|q_{v}(y) T v(y)\right| \leq 2 c F_{s}(y, \ln (|y|+1)) .
$$

Let $q_{0}(y)=2 c F_{s}(y, \ln (|y|+1))$ and let $Q_{0}$ be given as in (4.1). It follows that

$$
q_{v}(\cdot) T v(\cdot) \in Q_{0}
$$

and

$$
\left\{\frac{1}{h(\cdot)} \int_{D} G_{D}(\cdot, y) h(y) q_{v}(y) T v(y) d y: v \in \Lambda\right\} \subseteq G_{D}^{h}\left[Q_{0}\right] .
$$

We thus have by Proposition 4.1 that $T \Lambda$ is a relatively compact set in $\mathcal{P}(D)$. We shall prove the continuity of $T$ in $\Lambda$ in the supremum norm. Let $v_{n} \rightarrow v$ in $\Lambda$ as $n \rightarrow \infty$. Since $\tau<\infty$ for $P^{x}$-almost Brownian paths, we have by the continuity of function $f$ and the bounded convergence theorem that for almost every Brownian path:

$$
\int_{0}^{\tau_{D}} \frac{f\left(X_{t}, v_{n}\left(X_{t}\right) h\left(X_{t}\right)\right)}{2 v_{n}\left(X_{t}\right) h\left(X_{t}\right)} d t \rightarrow \int_{0}^{\tau_{D}} \frac{f\left(X_{t}, v\left(X_{t}\right) h\left(X_{t}\right)\right)}{2 v\left(X_{t}\right) h\left(X_{t}\right)} d t .
$$

Since $\left\{v_{n}\right\} \subset \Lambda$, each term of the sequence in (4.19) is bounded by

$$
\int_{0}^{\tau_{D}} F_{s}\left(X_{t}, 2 \eta c \ln \left(\left|X_{t}\right|+1\right)\right) d t .
$$

By (4.14) and Khas'minskii's lemma

$$
E_{h}^{x}\left[e^{\int_{0}^{\tau_{0} D} F_{s}\left(X_{t}, 2 \eta c \ln \left(\left|X_{t}\right|+1\right)\right) d t}\right] \leq 2<\infty,
$$

and hence it follows from (4.19), (4.20), and the dominated convergence theorem that $\forall x \in D$

$$
E_{h}^{x}\left[e^{\int_{0}^{\tau} D q_{v_{n}}\left(X_{t}\right) d t}\right] \rightarrow E_{h}^{x}\left[e^{\int_{0}^{\tau} D q_{v}\left(X_{t}\right) d t}\right] .
$$


Thus by (4.5)

$$
T v_{n}(x) \rightarrow T v(x)
$$

as $n \rightarrow \infty$. We have proved that $T \Lambda$ is a relatively compact family, therefore the pointwise convergence in (4.21) implies the uniform convergence, namely, $\left\|T v_{n}-T v\right\| \rightarrow 0$ as $n \rightarrow \infty$. Thus, we proved that $T$ is a compact and continuous mapping from $\Lambda$ to itself. By the definition of $\Lambda, \Lambda$ is obviously a nonempty, closed, bounded, and convex set in $\mathcal{P}(D)$. Hence by the Schauder fixed-point theorem, there exists a function $v \in \Lambda$ such that

$$
T v=v .
$$

It follows from (4.16) and (4.22) that

$$
v(x)=c+\frac{1}{h(x)} \int_{D} G_{D}(x, y) h(y) q_{v}(y) v(y) d y .
$$

Now let

$$
u(x)=h(x) v(x) .
$$

Since $v \geq c e^{-\frac{1}{2}}$ and $h>0$ in $D, u>0$ in $D$. By (4.6), (4.23), and (4.24), we have

$$
u(x)=\operatorname{ch}(x)+\frac{1}{2} \int_{D} G_{D}(x, y) f(y, u(y)) d y .
$$

Since $h$ is harmonic in $D$, applying $\Delta$ to both sides of (4.25) and using $\Delta\left(G_{D} f\right)=$ $-2 f$, we have

$$
\begin{aligned}
\Delta u(x) & =\frac{1}{2} \Delta\left[\int_{D} G_{D}(x, y) f(y, u(y)) d y\right] \\
& =-f(x, u(x)) .
\end{aligned}
$$

By the property of $h$ and the boundedness of $v$, we have

$$
\lim _{x \rightarrow \partial D} u(x)=0
$$

and

$$
\lim _{|x| \rightarrow \infty} \frac{u(x)}{\ln |x|}=\lim _{|x| \rightarrow \infty} \frac{v(x) h(x)}{\ln |x|}=c .
$$

We thus complete the proof.

\section{REFERENCES}

1. S. Axler, P. Bourdon,W. Ramey, "Harmonic Function Theory ", Springer-Verlag, 1992. MR 93f:31001

2. K. L. Chung, Z. Zhao, “From Brownian Motion to Schrödinger's Equation”, Springer, Berlin, 1995. MR 96f: 60140

3. J. B. Conway, "A Course in Functional Analysis- Second Edition", Springer-Verlag, 1990. MR 91e:46001

4. M. Cranston, E. Fabes, Z. Zhao, "Conditional gauge and potential theory for the Schrödinger Operators.”, Trans. Am. Math. Soc.307,(1983), 174-194. MR 90a:60135

5. F. Gesztesy, Z. Zhao, "On Positive Solutions of Critical Schrödinger Operators in Two Dimensions", Jour. of Func. Analy. 127(1995), 235-256. MR 96a:35037

6. I. W. Herbst, Z. Zhao, “ Green's functions for the Schrödinger equation with shortrange potentials.", Duke Math. J. 59 (1989), 475-519. MR 91a:35053 
7. C. Kenig, W. M. Ni, "An exterior Dirichlet problem with applications to some nonlinear equations arising in geometry", Amer.J.Math. 106(1984),689-702. MR 85j:35072

8. W.-M. Ni, "On the elliptic equation $\Delta u+K(x) u^{(n+2) /(n-2)}=0$, its generalizations and applications in geometry ", Indiana Univ. Math. J., 31(1995), 493-529.

9. S. Port and C. Stone, "Brownian Motion and Classical Potential Theory", Probab. Math. Statist., Academic Press, New York, 1978. MR 58:11459

10. Z. Zhao, "The Local Feynman-Kac Semigroup", J. Syst. Sci. Math. Sci.,2(4) (1982), 314-326. MR 87k:60190

11. Z. Zhao, "Green Functions for Schrödinger operator and conditioned Feynman-Kac gauge",J. Math. Anal. Appl., 116(1986), 309-334. MR 88f:60142

12. Z. Zhao, "Green Functions and conditioned gauge theorem for a 2-dimensional domain", Seminar on Stochastic Processes, Birkhauser, Boston, 1987, 283-294. MR 91e:35079

13. Z. Zhao, "On the Existence of Positive Solutions of Nonlinear Elliptic Equations-A probabilistic Potential Theory Approach", Duke Math. J., 69(1993),247-258. MR 94c:35090

14. Z. Zhao, "Positive Solutions of Nonlinear Second Order Ordinary Differential Equations", Proc.AMS, 121(1994), 465-469. MR 94h:34012

Department of Mathematics, University of Missouri, Columbia, Missouri 65211

Current address, U. Ufuktepe: Akdeniz Universitesi, Fen-Edebiyat Fakultesi, Matematik Bolumu, 07058 Antalya, Turkey

E-mail address: uunal@pascal.sci.akdeniz.edu.tr

E-mail address: mathzz@mizzou1.missouri.edu 